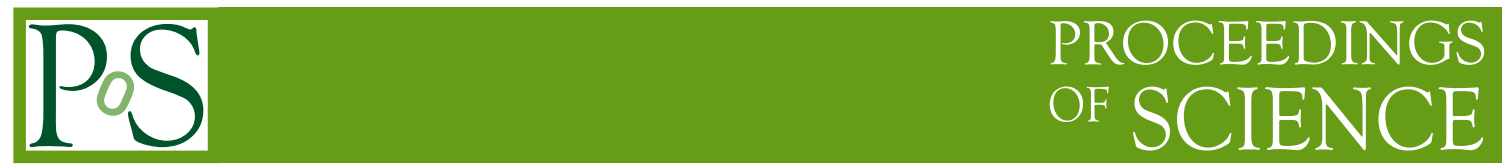

\title{
Review of LHCb Heavy-Quark and Quarkonia Results
}

\section{Zhenwei YANG*广}

Key Laboratory of Particle \& Radiation Imaging (Tsinghua University), Ministry of Education; Center for High Energy Physics, Department of Engineering Physics, Tsinghua University, Beijing, China

E-mail: yangzhw@mail.tsinghua.edu.cn, zhenwei.yang@cern.ch

Recent results of heavy-quark and quarkonia from the LHCb experiment are presented, focusing on the production cross-sections of $J / \psi$ and $\Upsilon$ mesons at $\sqrt{s}=8 \mathrm{TeV}$, the production ratios of $\chi_{c}$ mesons at $\sqrt{s}=7 \mathrm{TeV}$ using converted photons, and the polarisation of prompt $J / \psi$ mesons at $\sqrt{s}=7 \mathrm{TeV}$.

The European Physical Society Conference on High Energy Physics

18-24 July, 2013

Stockholm, Sweden

\footnotetext{
${ }^{*}$ Speaker.

${ }^{\dagger}$ for the LHCb collaboration
} 


\section{Introduction}

The studies of heavy-quark and quarkonia in $p p$ collisions are important since they provide essential tests of the QCD models. e.g., the non-relativistic QCD (NRQCD) [1] and the coloursinglet model (CSM) [2]. Great success has been achieved to describe differential production cross-sections of heavy quarkonia [3-5]. However, some open issues still exist, one well-known example is that these theoretical models failed to reproduce the polarisation of $J / \psi$ and $\psi(2 S)$ mesons measured by CDF [6]. Therefore, precise measurements of heavy-quark and quarkonia in $p p$ collisions are necessary for a better understanding of QCD, and provide essential constraints and inputs for theoretical models. This note presents recent results in this field from the LHCb experiment [7], based on the data samples taken in 2011 and 2012, focusing on the production of heavy quarkonia $[8,9]$, and the polarisation of prompt $J / \psi$ mesons [10]. Many other results are not mentioned due to the limit of pages.

\section{Productions of heavy quarkonia}

Following the previous measurements at $\sqrt{s}=7 \mathrm{TeV}$ and $2.76 \mathrm{TeV}$ [3-5], the double-differential production cross-sections for $J / \psi$ and $\Upsilon$ mesons at $\sqrt{s}=8 \mathrm{TeV}$ are measured as functions of the rapidity $y$ and the transverse momentum $p_{\mathrm{T}}$ [8], using the data collected in early 2012 with $18 \mathrm{pb}^{-1}$ $\left(51 \mathrm{pb}^{-1}\right)$ for $J / \psi(\Upsilon)$ mesons. Figure 1 shows the invariant mass distributions for $J / \psi$ and $\Upsilon$ mesons, with clean signal peaks and low backgrounds. The prompt $J / \psi$ mesons and $J / \psi$ mesons from $b$ hadron decays are well separated by combined fits to the invariant mass and the pseudo-proper time $t_{z}$ [8]. The integrated production cross-sections for prompt $J / \psi$ mesons and $J / \psi$ from $b$ are

$$
\begin{aligned}
& \sigma\left(\text { prompt } J / \psi, p_{\mathrm{T}}<14 \mathrm{GeV} / c, 2.0<y<4.5\right)=10.94 \pm 0.02 \pm 0.79 \mu \mathrm{b}, \\
& \sigma\left(J / \psi \text { from } b, p_{\mathrm{T}}<14 \mathrm{GeV} / c, 2.0<y<4.5\right)=1.28 \pm 0.01 \pm 0.11 \mu \mathrm{b} .
\end{aligned}
$$

The measured integrated production cross-sections for $\Upsilon(n S)$ time $\mathscr{B}^{n S}$ in the range $2.0<y<4.5$ and $p_{\mathrm{T}}<15 \mathrm{GeV} / c$ are

$$
\begin{aligned}
& \sigma(p p \rightarrow \Upsilon(1 S) X) \times \mathscr{B}^{1 S}=3.241 \pm 0.018 \pm 0.231 \mathrm{nb} \\
& \sigma(p p \rightarrow \Upsilon(2 S) X) \times \mathscr{B}^{2 S}=0.761 \pm 0.008 \pm 0.055 \mathrm{nb}, \\
& \sigma(p p \rightarrow \Upsilon(3 S) X) \times \mathscr{B}^{3 S}=0.369 \pm 0.005 \pm 0.027 \mathrm{nb} .
\end{aligned}
$$

In both cases the first uncertainty is statistical and the second one systematic.

The left plot of Fig. 2 shows the differential production cross-sections for prompt $J / \psi$ mesons as a function of $p_{\mathrm{T}}$ with $2.0<y<4.5$. The theoretical predictions shown in this plot are for direct $J / \psi$ meson production. The next-to-leading order (NLO) NRQCD model provides a prediction in good agreement with the data $[11,12]$. Considering the contribution of feed-down from higher charmonium states, the prediction of the NNLO* CSM also provide a reasonable description of the data, where NNLO* indicates that the calculations at the next-to-next-leading order are incomplete and some logarithmic terms are neglected [13]. However, the NLO CSM calculation underestimates the production cross-section by an order of magnitude [14]. The results for $J / \psi$ from $b$ are compared in the right plot of Fig. 2 to the calculations based on the FONLL formalism [15, 16]. As already 

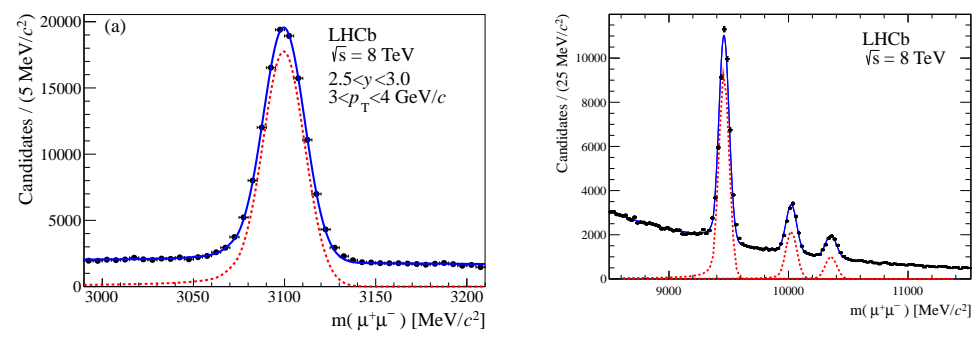

Figure 1: Distributions of dimuon invariant mass for (left) $J / \psi$ mesons with $3.0<p_{\mathrm{T}}<4.0 \mathrm{GeV} / c$ and $2.5<y<3.0$, and (right) $\Upsilon$ mesons with $p_{\mathrm{T}}<15 \mathrm{GeV} / c$ and $2.0<y<4.5$.

observed in $J / \psi$ production measurements at $\sqrt{s}=7 \mathrm{TeV}$ [3] and at $\sqrt{s}=2.76 \mathrm{TeV}$ [5], good agreements are shown both for the production cross-section as a function of $p_{\mathrm{T}}$ and that as a function of $y$ at $\sqrt{s}=8 \mathrm{TeV}$.
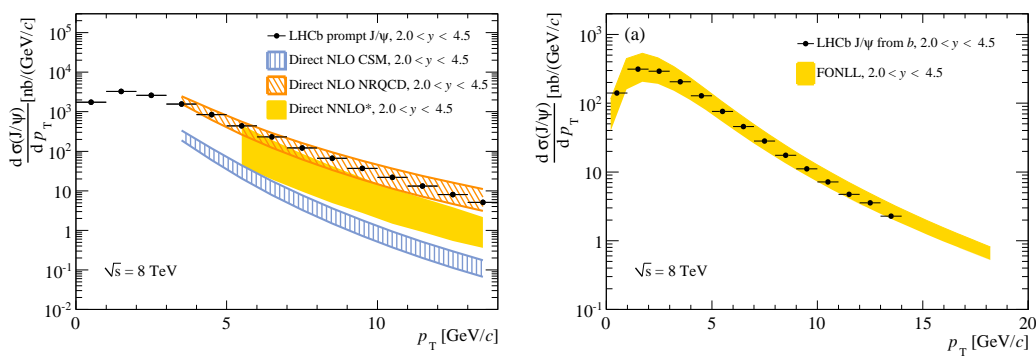

Figure 2: Differential production cross-section for (left) prompt $J / \psi$ mesons and (right) $J / \psi$ from $b$ as a function of $p_{\mathrm{T}}$. Theoretical predictions for direct $J / \psi$ mesons [11-14] are superposed in the left plot, and the prediction of FONLL $[15,16]$ is superposed in the right plot.

Figure 3 shows the production cross-sections as functions of $p_{\mathrm{T}}$ time the dimuon branching fractions $\mathscr{B}^{n S} \equiv \mathscr{B}\left(\Upsilon(n S) \rightarrow \mu^{+} \mu^{-}\right)$for the three $\Upsilon$ meson states, together with the theoretical predictions of NLO CSM [14] and NNLO* CSM [13]. The NNLO* CSM shows a modest agreement with the data, especially for $\Upsilon(3 S)$ mesons, which are expected to suffer less from feed-down. Similar to the case of the prompt $J / \psi$ production, NLO CSM greatly underestimates the production cross-sections.
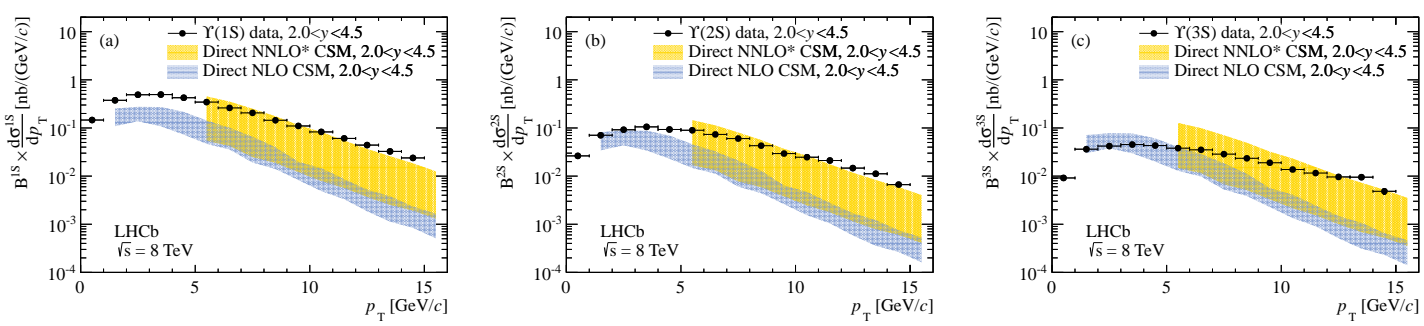

Figure 3: Differential production cross-sections times dimuon branching fractions for (a) $\Upsilon(1 S)$, (b) $\Upsilon(2 S)$, and (c) $\Upsilon(3 S)$ mesons as functions of $p_{\mathrm{T}}$, compared to theoretical predictions for direction production in NLO CSM [14] and NNLO* CSM [13].

It is also important to measure the production of $\chi_{c}$ states. NRQCD calculations with both colour-singlet and colour-octet contributions taken into account give a raito of the $\chi_{c 2}$ to $\chi_{c 1}$ 
production cross-sections, which is different to the caluclation of CSM. The radiative decay $\chi_{c} \rightarrow$ $J / \psi \gamma$ substantially contributes to the prompt $J / \psi$ production [17], and can also have a significant impact on the polarisation of prompt $J / \psi$ [10]. The ratio of prompt $\chi_{c 2}$ to $\chi_{c 1}$ production crosssections $\sigma\left(p p \rightarrow \chi_{c 2} X\right) / \sigma\left(p p \rightarrow \chi_{c 1} X\right)$ at $\sqrt{s}=7 \mathrm{TeV}$ was first measured using calorimetric photons, using a data sample of $36 \mathrm{pb}^{-1}$ collected by the LHCb detector in 2010 [17]. This ratio is updated by a measurement with photons converted into $e^{+} e^{-}$before entering the $\mathrm{LHCb}$ magnet, using a data sample of $1.0 \mathrm{fb}^{-1}$ taken in 2011 [9]. Although the efficiency of converted photons is rather low, the resolution of the invariant mass of $J / \psi \gamma$ is greatly improved, as shown in the left plot of Fig. 4. An evidence of $\chi_{c 0}$ signal is first observed in hadron colliders with a statistical significance of $4.3 \sigma$. The extracted signal yield in the range $4<p_{\mathrm{T}}^{J / \psi}<20 \mathrm{GeV} / c$ is $N\left(\chi_{c 0}\right)=705 \pm 163$,

The right plot shows the ratio of $\chi_{c 2}$ to $\chi_{c 1}$ production cross-sections as a function of $p_{\mathrm{T}}^{J / \psi}$, together with a comparison to the calculations in NLO NRQCD [18] and in LO NRQCD [19]. Both calculations provide a reasonable description of the data, particularly in high $p_{\mathrm{T}}^{J / \psi}$ region. The ratio of $\chi_{c 0}$ to $\chi_{c 2}$ production cross-sections, integrated over $4<p_{\mathrm{T}}^{J / \psi}<20 \mathrm{GeV} / c$, is

$$
\begin{aligned}
& \sigma\left(\chi_{c 0}\right) / \sigma\left(\chi_{c 2}\right)=1.19 \pm 0.27(\text { stat }) \pm 0.29(\text { syst }) \pm 0.16\left(p_{\mathrm{T}} \text { model }\right) \pm 0.09(\mathscr{B}), \\
& \sigma\left(\chi_{c 2}\right) / \sigma\left(\chi_{c 1}\right)=0.787 \pm 0.014(\text { stat }) \pm 0.034(\text { syst }) \pm 0.051\left(p_{\mathrm{T}} \text { model }\right) \pm 0.047(\mathscr{B}),
\end{aligned}
$$

where the first uncertainty is statistical, the second is systematic, the third is due to the $p_{\mathrm{T}}$ spectrum of the chosen model, and the fourth is due to the branching fraction uncertainty.
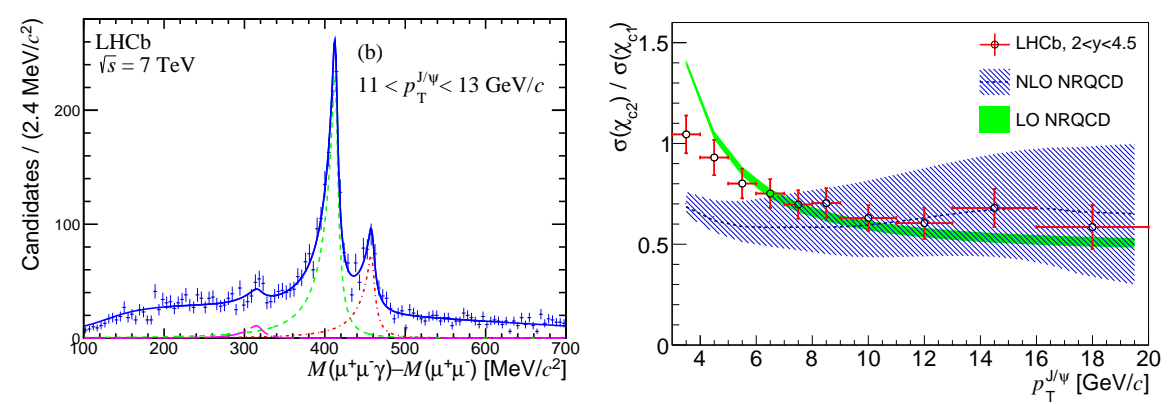

Figure 4: (left) Distribution of $\Delta M \equiv M\left(\mu^{+} \mu^{-} \gamma\right)-M\left(\mu^{+} \mu^{-}\right)$for $\chi_{c}$ candidates with $11<p_{\mathrm{T}}^{J / \psi}<13 \mathrm{GeV} / c$. (right) Ratio of $\chi_{c 2}$ to $\chi_{c 1}$ production cross-sections at $\sqrt{s}=7 \mathrm{TeV}$ as a function of $p_{\mathrm{T}}^{J / \psi}$ for $2.0<y<4.5$, together with the calculations in NLO NRQCD [18] and in LO NRQCD [19].

\section{Polarisation measurements of prompt $J / \psi$ mesons}

A measurement of prompt $J / \psi$ polarisation in $p p$ collisions at $\sqrt{s}=7 \mathrm{TeV}$ is performed, using a dataset of an integrated luminosity of $0.37 \mathrm{fb}^{-1}$ collected by the LHCb detector in early 2011 [10]. The polarisation parameters are determined as functions of $p_{\mathrm{T}}$ and $y$ by a full angular analysis of the decay $J / \psi \rightarrow \mu^{+} \mu^{-}$.

Figure 5 shows the measured polarisation parameter $\lambda_{\theta}$ for prompt $J / \psi$ mesons in the helicity frame as a function of $p_{\mathrm{T}}$ in the range $2.5<y<4.0$, accompanied by the comparison with (left) the ALICE result [20] and (right) theoretical predictions [21-23]. The ALICE result is for inclusive 
$J / \psi$ mesons, and the uncertainties are rather large. The measurements of ALICE and LHCb are compatible. The right plot of Fig. 5 shows that our measurement disagrees with the calculations in NLO CSM [21]. For the NLO NRQCD calculations different schemes are used in the three Refs. [21-23] to evaluate the non-perturbative matrix elements. The results show a better agreement with the NLO NRQCD calculations in Ref. [23] than in Ref. [21,22]. However, one should note that neither NLO CSM nor NLO NRQCD calculations in Ref. [21] includes the effect of feed-down from excited charmonium states, while the feed-down from $\chi_{c}$ and $\psi(2 S)$ mesons are taken into account in the NLO NRQCD calculations in Refs. [22,23].
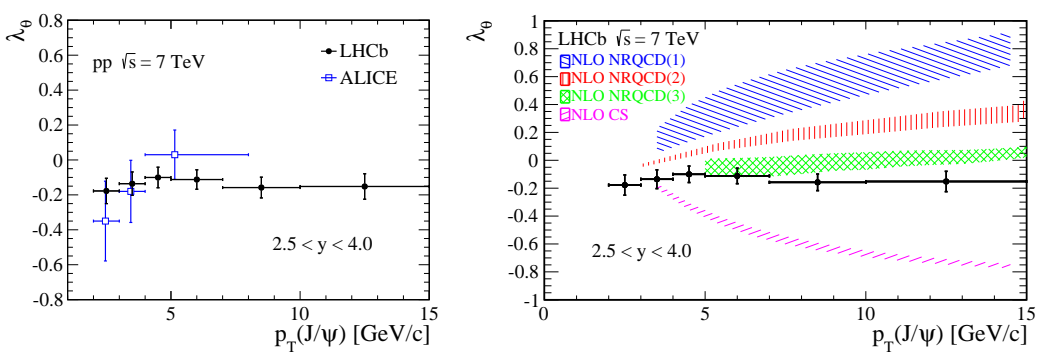

Figure 5: Polarisation parameter $\lambda_{\theta}$ for prompt $J / \psi$ mesons in the helicity frame, together with (left) the ALICE results [20] and (right) theoretical predictions [21-23].

With the measured polarisation, the prompt $J / \psi$ production cross-sections at $\sqrt{s}=7 \mathrm{TeV}$ is updated, which was previously measured in 2011 [3] with large uncertainties due to the unknown polarisation of $J / \psi$ mesons. The updated integrated cross-section in the range $p_{\mathrm{T}}<14 \mathrm{GeV} / c$ and $2.0<y<4.5$ is $\sigma_{\text {prompt }}=9.46 \pm 0.04 \pm 0.53_{-1.10}^{+0.86} \mu \mathrm{b}$, where the first uncertainty is statistical, the second systematic, and the third asymmetric uncertainties originate from the unknown polarisation of $J / \psi$ mesons with $p_{\mathrm{T}}<2 \mathrm{GeV} / c$.

\section{Summary}

The $\mathrm{LHCb}$ experiment has presented a lot of measurements of heavy-quark and quarkonia using the data of $p p$ collisions at $7 \mathrm{TeV}$ and $8 \mathrm{TeV}$ accumulated in 2011 and 2012, among which some recent results are summarised in this note. The production cross-sections of $J / \psi$ and $\Upsilon$ mesons at $\sqrt{s}=8 \mathrm{TeV}$ are measured using the dimuon final state [8]. The ratio of $\chi_{c 2}$ to $\chi_{c 1}$ production cross-sections is measured at $\sqrt{s}=7 \mathrm{TeV}$ using converted photons [9]. The polarisation of prompt $J / \psi$ mesons is determined using a data sample of $p p$ collisions at $\sqrt{s}=7 \mathrm{TeV}$ [10].

\section{References}

[1] W. Caswell and G. Lepage, Effective Lagrangians for bound state problems in QED, QCD, and other field theories, Phys.Lett. B167 (1986) 437.

[2] R. Baier and R. Ruckl, Hadronic Collisions: A Quarkonium Factory, Z.Phys. C19 (1983) 251.

[3] LHCb collaboration, R. Aaij et al., Measurement of $J / \psi$ production in pp collisions at $\sqrt{s}=7 \mathrm{TeV}$, Eur. Phys. J. C71 (2011) 1645 [ arXiv:1103.0423 ].

[4] LHCb collaboration, R. Aaij et al., Measurement of $\Upsilon$ production in pp collisions at $\sqrt{s}=7 \mathrm{TeV}$, Eur. Phys. J. C72 (2012) 2025 [ arXiv:1202.6579 ]. 
[5] LHCb collaboration, R. Aaij et al., Measurement of $J / \psi$ production in pp collisions at $\sqrt{s}=2.76 \mathrm{TeV}$, JHEP 02 (2013) 41 [ arXiv:1212.1045 ].

[6] CDF collaboration, A. Abulencia et al., Polarization of $J / \psi$ and $\psi(2 S)$ mesons produced in $p \bar{p}$ collisions at $\sqrt{s}=1.96 \mathrm{TeV}$, Phys.Rev.Lett. 99 (2007) 132001 [ arXiv:0704.0638 ].

[7] LHCb collaboration, A. A. Alves Jr. et al., The LHCb detector at the LHC, JINST 3 (2008) S08005.

[8] LHCb collaboration, R. Aaij et al., Production of $J / \psi$ and $\Upsilon$ mesons in pp collisions at $\sqrt{s}=8 \mathrm{TeV}$, JHEP 06 (2013) 64 [ arXiv:1304.6977 ].

[9] LHCb collaboration, R. Aaij et al., Measurement of the relative rate of prompt $\chi_{c 1}, \chi_{c 2}$ and $\chi_{c 0}$ production at $\sqrt{s}=7 \mathrm{TeV}$, JHEP 10 (2013) 115 [ arXiv:1307.4285 ].

[10] LHCb collaboration, R. Aaij et al., Measurement of $J / \psi$ polarization in pp collisions at $\sqrt{s}=7 \mathrm{TeV}$, arXiv:1307.6379. to appear in Eur. Phys. J. C.

[11] Butenschön, Mathias and Kniehl, Bernd A., World data of $J / \psi$ production consolidate NRQCD factorization at NLO, Phys.Rev. D84 (2011) 051501 [ arXiv:1105.0820 ].

[12] Butenschön, Mathias and Kniehl, Bernd A., Reconciling J/ $\psi$ production at HERA, RHIC, Tevatron, and LHC with nonrelativistic QCD factorization at next-to-leading order, Phys.Rev.Lett. 106 (2011) 022003 [ arXiv:1009.5662 ].

[13] J. Lansberg, On the mechanisms of heavy-quarkonium hadroproduction, Eur.Phys.J. C61 (2009) 693-703 [ arXiv:0811.4005 ].

[14] J. M. Campbell, F. Maltoni, and F. Tramontano, QCD corrections to J/ $\psi$ and $\Upsilon$ production at hadron colliders, Phys.Rev.Lett. 98 (2007) 252002 [ arXiv:hep-ph/0703113 ].

[15] M. Cacciari, S. Frixione, N. Houdeau, M. L. Mangano, P. Nason, et al., Theoretical predictions for charm and bottom production at the LHC, JHEP 10 (2012) 137 [ arXiv:1205.6344 ].

[16] M. Cacciari, M. Greco, and P. Nason, The $p_{\mathrm{T}}$ spectrum in heavy flavor hadroproduction, JHEP 9805 (1998) 007 [ arXiv:hep-ph/9803400 ].

[17] LHCb collaboration, R. Aaij et al., Measurement of the ratio of prompt $\chi_{c}$ to $J / \psi$ production in $p p$ collisions at $\sqrt{s}=7 \mathrm{TeV}$, Phys. Lett. B718 (2012) 431 [ arXiv:1204.1462 ].

[18] Y.-Q. Ma, K. Wang, and K.-T. Chao, QCD radiative corrections to $\chi_{c J}$ production at hadron colliders, Phys.Rev. D83 (2011) 111503 [ arXiv:1002.3987].

[19] A. Likhoded, A. Luchinsky, and S. Poslavsky, Hadronic production of $\chi_{c}$-mesons at LHC, arXiv:1305.2389.

[20] ALICE collaboration, B. Abelev et al., $J / \psi$ polarization in pp collisions at $\sqrt{s}=7 \mathrm{TeV}$, Phys.Rev.Lett. 108 (2012) 082001 [ arXiv:1111.1630 ].

[21] Butenschön, Mathias and Kniehl, Bernd A., J/ $\psi$ production in NRQCD: a global analysis of yield and polarization, Nucl.Phys.Proc.Suppl. 222-224 (2012) 151-161 [ arXiv:1201.3862 ].

[22] B. Gong, L.-P. Wan, J.-X. Wang, and H.-F. Zhang, Polarization for prompt J/ $\psi, \psi(2 S)$ production at the Tevatron and LHC, Phys.Rev.Lett. 110 (2013) 042002 [ arXiv:1205.6682 ].

[23] K.-T. Chao, Y.-Q. Ma, H.-S. Shao, K. Wang, and Y.-J. Zhang, J/ $\psi$ polarization at hadron colliders in nonrelativistic QCD, Phys.Rev.Lett. 108 (2012) 242004 [ arXiv:1201.2675 ]. 